\title{
Interrelationship of dipeptidyl peptidase IV (DPP4) with the development of diabetes, dyslipidaemia and nephropathy: a streptozotocin-induced model using wild-type and DPP4-deficient rats
}

\author{
Yasushi Kirino" ${ }^{1}$, Youichi Sato ${ }^{1}$, Takayuki Kamimoto², Kazuyoshi Kawazoe², Kazuo Minakuchi \\ and Yutaka Nakahori ${ }^{1}$ \\ Departments of ${ }^{1}$ Human Genetics and Public Health and ${ }^{2}$ Clinical Pharmacy, Institute of Health Biosciences, The University of Tokushima Graduate School, \\ The University of Tokushima, 3-18-15 Kuramoto-Cho, Tokushima 770-8503, Japan \\ (Correspondence should be addressed to Y Nakahori; Email: nakahori@basic.med.tokushima-u.ac.jp)
}

\begin{abstract}
We examined the role of dipeptidyl peptidase IV (DPP4) in the development of diabetes, dyslipidaemia and renal dysfunction induced by streptozotocin (STZ). F344/ DuCrlCrlj rats, which lack DPP4 activity, and wild-type rats were treated with STZ. Plasma DPP4 activity and biochemical parameters were measured until 42 days after STZ treatment. At the end of the experiment, renal function and DPP4 expressions of the kidney, liver, pancreas and adipose tissues were determined. Increases in blood glucose, cholesterol and triglycerides were evoked by STZ in both rat strains; however, the onset of hyperglycaemia was delayed in DPP4-deficient rats as compared with wild-type rats. By contrast, more severe dyslipidaemia was observed in DPP4deficient rats than in wild-type rats after STZ treatment. Plasma DPP4 activity increased progressively with time after
\end{abstract}

STZ treatment in wild-type rats. The kidney of wild-type rats showed decreased DPP4 activity with increased Dpp 4 mRNA after STZ treatment. In addition, kidney weight, serum creatinine and excreted amounts of urinary protein, glucose and DPP4 enzyme were enhanced by STZ. DPP4-deficient rats showed increased serum creatinine in accordance with decreased creatinine clearance as compared with wild-type rats after STZ treatment. In conclusion, plasma DPP4 activity increased after STZ treatment, positively correlating to blood glucose. DPP4-deficient rats were resistant to developing diabetes, while susceptible to dyslipidaemia and reduction of glomerular filtration rate by STZ. DPP4 activation may be responsible for hyperglycaemia, lipid metabolism and preservation of renal function.

Journal of Endocrinology (2009) 200, 53-61

\section{Introduction}

Dipeptidyl peptidase IV (DPP4, EC3.4.14.5) exists on the surface of various types of cells, such as kidney, liver, small intestine, pancreas, and in a soluble form in plasma (Mentlein 1999). DPP4 is a serine protease, which cleaves the penultimate $L$-proline or $L$-alanine at the $N$-terminus of several polypeptides, such as glucagon-like peptide 1 (GLP-1) and glucose-dependent insulinotropic polypeptide (GIP; De Meester et al. 2000). The major incretin hormones, GLP-1 and GIP, are gastrointestinal peptides involved in the regulation of postprandial nutrient homeostasis (Green et al. 2004a, Holst 2006). These peptides augment nutrient-induced insulin release from pancreatic $\beta$-cells in a glucose-dependent fashion (Green et al. 2004a,b, Holst 2006). These are also reported to be associated with insulin biosynthesis, proliferation of pancreatic $\beta$-cells and inhibition of food intake (Pospisilik et al. 2003, Holst 2006). Abundant studies in both human and animal models have established DPP4 inhibition, followed by GLP-1 elevation, as a promising therapeutic strategy for the treatment of diabetes (Deacon et al. 2002, Pospisilik et al. 2003, Flock et al. 2007, Fonseca et al. 2007, Mikhail 2008). The Food and Drug Administration and the European Medicines Agency recently approved a DPP4 inhibitor for use in type 2 diabetic patients. However, it remains unclear whether DPP4 activity is correlated to the onset or severity of diabetes and diabetic complications or not. In an in vitro study, DPP4 activity and mRNA expression were enhanced by exposure of human glomerular endothelial cells to high glucose (Pala et al. 2003). On the other hand, in various published clinical reports over the last decade, circulating DPP4 activity has been reported to be both increased (Mannucci et al. 2005, Ryskjaer et al. 2006) and decreased (Meneilly et al. 2000, McKillop et al. 2008) in diabetic patients. Additionally, it has been reported that the degree of plasma DPP4 activity was associated with obesity (Lugari et al. 2004), gender (Durinx et al. 2001) and aging (Meneilly et al. 2000, Durinx et al. 2001, Ryskjaer et al. 2006). Moreover, several reports, including clinical studies, have confirmed that widely used anti-diabetic agents, metformin (Lenhard et al. 2004, Lindsay et al. 2005, Green et al. 2006) and pioglitazone 
(Lenhard et al. 2004), reduced the levels of circulating DPP4 activity in vivo. Considering these clinical findings, it may be complicated to define the correlation of DPP4 activity to the severity of hyperglycaemia because of the diverse clinical background; therefore, experimental diabetic models with unified conditions can support to identify whether DPP4 activation is physiologically involved or not.

F344/DuCrlCrlj rats, which are a substrain of the inbred Fischer 344 (F344) strain maintained at Charles River Japan (Osaka, Japan) lack DPP4 enzyme activity (Thompson et al. 1991, Erickson et al. 1992, Mitani et al. 2002, Yasuda et al. 2002). F344/DuCrlCrlj rats express Dpp 4 mRNA but lack DPP4 activity due to the translation of abnormal isoforms that fail to be processed to the biologically active mature glycosylated enzyme (Thompson et al. 1991, Erickson et al. 1992). F344/DuCrlCrlj rats have reduced GLP-1 degradation ability, which leads to higher levels of plasma active GLP-1, as compared with wildtype F344/Jcl rats (Yasuda et al. 2002). Accordingly, F344/ DuCrlCrlj rats, compared with wild-type F344/Jcl rats, can efficiently assess the responsibility of DPP4 activity for the development of diabetes and diabetic complications such as nephropathy. A couple of studies have previously reported that high-fat diet-induced insulin resistance and glucose intolerance were less prevalent in $\mathrm{F} 344 / \mathrm{DuCrlCrlj}$ rats than wild-type F344/Jcl rats (Mitani et al. 2002, Yasuda et al. 2002); however, no data have determined the significance of DPP4 activity in insulin-dependent diabetes, dyslipidaemia and diabetic renal dysfunction.

In the present study, the changes in DPP4 expressions of plasma and several tissues (kidney, liver, pancreas and adipose tissue) were determined in a streptozotocin (STZ)-induced diabetic model. In addition, differences in the levels of blood glucose, insulin, cholesterol, triglycerides, creatinine and urinary parameters were determined between F344/Jcl (wildtype) and F344/DuCrlCrlj (DPP4-deficient) rats treated with or without STZ, to assess the importance of DPP4 activity in hyperglycaemia, lipid metabolism and renal function.

\section{Materials and Methods}

\section{Experimental design}

Male F344/Jcl (wild-type) and F344/DuCrlCrlj (DPP4deficient) rats aged 8 weeks were purchased from Clea Japan (Tokyo, Japan) and Charles River Japan (Osaka, Japan) respectively. All rats were housed in an air-conditioned room at $23 \pm 2{ }^{\circ} \mathrm{C}$ with $50 \pm 10 \%$ humidity under controlled lighting conditions (12h light: $12 \mathrm{~h}$ darkness cycle). The rats were fed a commercial diet (MF; Oriental Yeast, Tokyo, Japan) and had access to tap water ad libitum. At 9 weeks of age, rats were randomly divided into the following four groups: wild-type (control), $n=6$; wild-type (STZ), $n=10$; DPP4-deficient (control), $n=6$; and DPP4-deficient (STZ), $n=10$. Experimental diabetes was induced by a single i.p. injection of STZ (30 mg/kg; Wako Pure Chemical, Osaka,
Japan) in $0.05 \mathrm{M}$ citrate buffer ( $\mathrm{pH} 4.5$ ) after an overnight fast, and then the rats were re-fed. Control rats received an equivalent amount of citrate buffer. Before and $6 \mathrm{~h}, 1,3,14$, 28 and 42 days after injection of STZ or citrate buffer, blood samples were obtained from tail veins. Over a 24-hour period between days 35 and 37 , the rat was placed in a metabolic cage containing a urine collection funnel to measure urine excretion. All rats had access to food and water ad libitum while in the cages. Urine was collected for exactly $24 \mathrm{~h}$ and the volume was measured. Urine samples were stored at $-20^{\circ} \mathrm{C}$ until the measurement of several parameters. At the end of the experimental period, the right kidney, liver, pancreas and epididymal fat were immediately collected and weighed after the rats were killed under anaesthesia with urethane $(5 \mathrm{~g} / \mathrm{kg}$, intraperitoneally; Sigma). The collected tissues for DPP4 enzyme assay and the pancreas for insulin assay were immediately frozen and stored at $-80^{\circ} \mathrm{C}$. For purification of RNA from each tissue, the harvested samples were immediately cut into slices less than $5 \mathrm{~mm}$ thick, and the slices were stored in RNAlater RNA Stabilization Reagent (Qiagen) at $-20^{\circ} \mathrm{C}$. All animal care and treatments were conducted in accordance with the guidelines of the animal use and care committee of the University of Tokushima.

\section{Measurement of blood glucose, plasma insulin and lipid parameters}

After blood samples were obtained from tail veins, glucose concentrations were immediately measured using a glucose analyser (Glucose Pilot; Avenitir Biotech, Carlsbad, CA, USA). The collected blood samples were immediately centrifuged to separate plasma. Plasma insulin concentration was measured by ELISA using an Ultra-Sensitive Rat Insulin Kit (Morinaga Institute of Biological Science, Yokohama, Japan; Iwakura et al. 2005). The levels of cholesterol and triglycerides were measured using reagents manufactured by Wako Pure Chemical Industries.

\section{DPP4 enzyme assay in plasma}

DPP4 activity was determined by the cleavage rate of 7-amino-4-methylcoumarin (AMC) from the synthetic substrate $H$-glycyl-prolyl-AMC (Gly-Pro-AMC; Sigma), as described previously (Villhauer et al. 2003), with some modifications. Briefly, $5 \mu \mathrm{l}$ sample was mixed with $35 \mu 1$ assay buffer ( $25 \mathrm{mmol} / \mathrm{l}$ HEPES, $140 \mathrm{mmol} / \mathrm{l} \mathrm{NaCl}$, $80 \mathrm{mmol} / 1 \mathrm{MgCl}_{2}, 1 \%(\mathrm{w} / \mathrm{v})$ BSA (pH 7.8)). After 5-min preincubation at room temperature, the reaction was initiated by the addition of $40 \mu \mathrm{l}$ assay buffer containing $0.1 \mathrm{mmol} / \mathrm{l}$ substrate Gly-Pro-AMC. After incubation for $20 \mathrm{~min}$, fluorescence was determined using a spectrofluorometer (Tecan Infinite M200, Tecan Japan, Yokohama; excitation $380 \mathrm{~nm} /$ emission $460 \mathrm{~nm}$ ). The standard curve of free AMC was generated using $0-50 \mu \mathrm{mol} / 1$ solutions of AMC (Sigma). DPP4 activity in plasma was expressed as the amount of cleaved AMC per minute per $\mathrm{ml}(\mathrm{nmol} / \mathrm{min} / \mathrm{ml})$. 


\section{Immunohistochemistry}

The pancreas was dissected from surrounding tissue, fixed overnight in $10 \%(\mathrm{v} / \mathrm{v})$ formaldehyde and embedded in paraffin. Immunostaining was conducted according to the standard peroxidase reaction method. Briefly, sections were incubated with $3 \%(\mathrm{v} / \mathrm{v}) \mathrm{H}_{2} \mathrm{O}_{2}$ for $10 \mathrm{~min}$ to avoid nonspecific peroxidase reactions. Histological sections were immunostained for insulin using monoclonal anti-insulin (1:1000 dilution; Sigma) as the primary antibody and peroxidase-conjugated rabbit anti-mouse IgG (1:200 dilution; Sigma) as the secondary antibody. The sections were then incubated with 3,3'-diaminobenzidine tetrahydrochloride solution for $15 \mathrm{~min}$. After immunostaining, the sections were lightly counterstained with Mayer's hematoxylin, and immunoreactive cells were observed under a light microscope.

\section{Pancreatic insulin content}

Pancreatic insulin was extracted with the acid ethanol method $(0.18 \mathrm{mmol} / 1$ hydrochloric acid in $70 \%(\mathrm{v} / \mathrm{v})$ ethanol; Zhou et al. 2003), and then the insulin concentrations were determined (Ultra Sensitive Rat Insulin Kit; Morinaga Institute of Biological Science). Data were normalized by the protein concentration (Bradford Protein Assay; Bio-Rad Laboratories).

\section{DPP4 enzyme assay in kidney, liver, pancreas and epididymal fat}

Frozen tissues were homogenized in cold buffer $(25 \mathrm{mmol} / 1$ HEPES, $140 \mathrm{mmol} / 1 \mathrm{NaCl}, 80 \mathrm{mmol} / 1 \mathrm{MgCl}_{2}(\mathrm{pH} \mathrm{7} \cdot 8)$ ) containing $1 \%(\mathrm{v} / \mathrm{v})$ Triton X-100. Following homogenization, the samples were centrifuged at $1000 \mathrm{~g}$ for $10 \mathrm{~min}$ at $4{ }^{\circ} \mathrm{C}$. The supernatants were collected and centrifuged twice at $20000 \mathrm{~g}$ for $10 \mathrm{~min}$ at $4{ }^{\circ} \mathrm{C}$. The final supernatants were immediately used for DPP4 enzyme assay, which was described above. DPP4 activity in each tissue was expressed as the amount of cleaved AMC per minute per tissue weight (nmol/min/g tissue).

\section{Quantitative real-time PCR}

Total RNA was isolated using the RNeasy Lipid Tissue Mini Kit (Qiagen), according to the manufacturer's methods. cDNA was synthesized using $1 \mu \mathrm{g}$ total RNA and SuperScript III Reverse Transcriptase (Invitrogen). Real-time PCR was performed using SYBR Premix Ex Taq (Takara, Tokyo, Japan) on the AB 7500 real-time PCR system (Applied Biosystems, Foster City, CA, USA) with the following thermal cycling profile: initial denaturation at $95{ }^{\circ} \mathrm{C}$ for $10 \mathrm{~s}$ followed by 40 cycles of amplification (denaturation at $95^{\circ} \mathrm{C}$ for $10 \mathrm{~s}$, annealing at $60^{\circ} \mathrm{C}$ for $10 \mathrm{~s}$ and extension at $72{ }^{\circ} \mathrm{C}$ for $34 \mathrm{~s}$ ) and normalized to the expression of glyceraldehyde-3-phosphate dehydrogenase (GAPDH). The primers used in real-time PCR were the following: rat
DPP4 sense 5'-CTCCAGAGGACAACCTTGAC-3', antisense 5'-GGACAAGTGTGCTCTTGAGT-3'; rat GAPDH sense $5^{\prime}$-CTGAGAATGGGAAGCTGGTCAT-3' ${ }^{\prime}$, antisense 5'-TGGTGCAGGATGCATTGCT-3'.

\section{Urinary parameters and renal function}

Proteinuria was assessed by the Bradford method (Bradford Protein Assay Kit; Bio-Rad Laboratories). Urinary glucose concentrations were determined using a glucose analyser (Glucose Pilot; Avenitir Biotech). DPP4 activity in urine was determined using the same method as for the plasma DPP4 assay. Serum and urinary creatinine were measured by the Jaffé method using a commercial reagent (Wako Pure Chemical). Data are shown as urinary excretion $(\mathrm{ml} / 24 \mathrm{~h})$, protein excretion $(\mathrm{mg} / 24 \mathrm{~h})$, glucose excretion (nmol/24 h) and DPP4 excretion (nmol/min/24 h). Creatinine clearance (Ccr: $\mathrm{ml} / \mathrm{min} / \mathrm{kg}$ ) was used to estimate glomerular filtration rate (GFR).

\section{Data analysis}

All values are expressed as the means \pm s.E.M. Statistical significance was evaluated by Student's unpaired $t$-test for comparison between two groups using JMP (release 7.0; SAS Institute, Cary, NC, USA).

\section{Results}

Body weight, food intake, blood glucose, plasma insulin, cholesterol, triglycerides and DPP4 activity

No significant differences in body weight, food intake, blood glucose, insulin, cholesterol and triglycerides were found between F344/Jcl (wild-type) and F344/DuCrlCrlj (DPP4deficient) rats at 0 days under fasting (Fig. 1). The levels of blood glucose, cholesterol and triglycerides were not significantly different between these rat strains in the control group (not treated with STZ) at any points of the experiment (Fig. 1). However, the levels of plasma insulin in DPP4-deficient (control) rats were significantly lower than that in the wildtype (control) 6 h to 42 days after feeding (Fig. 1e and f). In STZtreated groups, limited body weight gain (Fig. 1a) and increased food intake (Fig. 1b) were observed as compared with the control in both rat strains. No significant difference in body weight was found between wild-type and DPP4-deficient rats treated with or without STZ, but food intake 7 days after STZ treatment in DPP4-deficient rats was significantly less than that in wild-type rats (Fig. 1b). Although marked hyperglycaemia and hypoinsulinaemia occurred after STZ injection in both rat strains, DPP4-deficient rats exhibited relatively lower blood glucose than wild-type rats in the early phase ( $6 \mathrm{~h}$ to 28 days) after STZ treatment despite no differences in insulin levels (Fig. 1c-f). Blood glucose levels in both rat strains reached a plateau (about $30 \mathrm{mmol} / \mathrm{l}$ ) 42 days after STZ treatment. Regarding the results of lipid parameters, STZ elevated the 

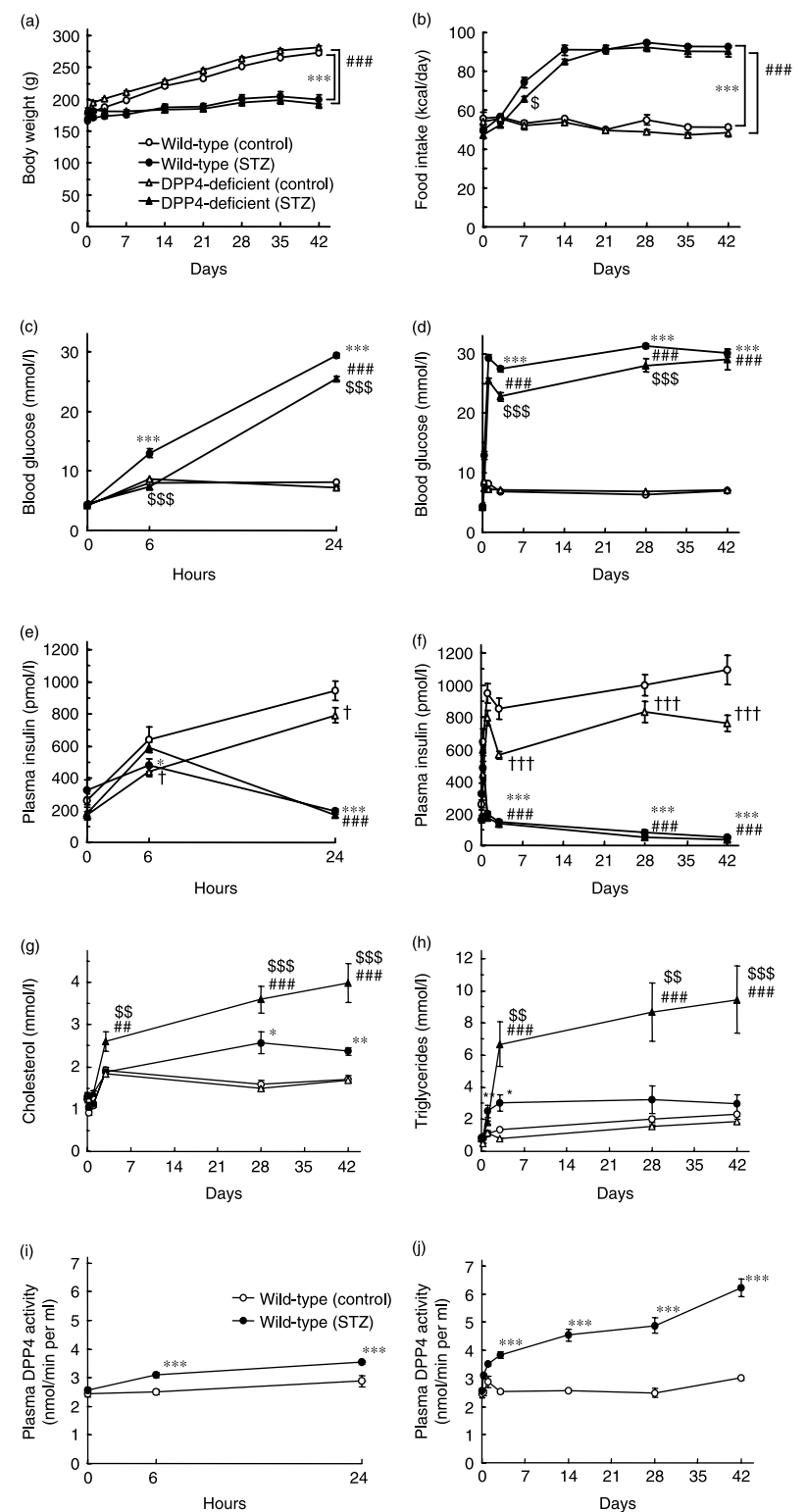

Figure 1 Changes in (a) body weight, (b) food intake, (c-d) blood glucose, (e-f) plasma insulin, (g) cholesterol, (h) triglycerides and (ij) plasma DPP4 activity in F344/Jcl (wild-type) and F344/DuCrlCrlj (DPP4-deficient) rats after i.p. injection with or without STZ $(30 \mathrm{mg} / \mathrm{kg})$. Data are shown as the means \pm s.E.M. $* P<0 \cdot 05$, ${ }^{* *} P<0 \cdot 01$ and ${ }^{* * *} P<0 \cdot 001$ for differences between wild-type (control) and wild-type (STZ). ${ }^{\# \#} P<0 \cdot 01$ and ${ }^{\# \#} P<0 \cdot 001$ for differences between DPP4-deficient (control) and DPP4-deficient (STZ). ${ }^{\dagger} P<0.05$ and ${ }^{+++} P<0.001$ for differences between wild-type (control) and DPP4-deficient (control). ${ }^{\$} P<0 \cdot 05,{ }^{\$ \$} P<0 \cdot 01$ and $\$ \$ P<0.001$ for differences between wild-type (STZ) and DPP4deficient (STZ). ${ }^{* * *}$ and ${ }^{\# \#}$ in Fig. 1 a are the values from 1 to 42 days respectively. ${ }^{* * *}$ and ${ }^{\# \#}$ in Fig. $1 \mathrm{~b}$ are the values from 7 to 42 days respectively. Open circles: wild-type (control) $n=6$; closed circles: wild-type (STZ) $n=10$; open triangles: DPP4-deficient (control) $n=6$; closed triangles: DPP4-deficient (STZ) $n=10$. levels of cholesterol (Fig. 1g) and triglycerides (Fig. 1h) to some extent in wild-type rats, exhibiting significanct differences in cholesterol levels at 28-42 days and triglycerides levels at 1-3 days respectively. In DPP4-deficient rats, however, both cholesterol and triglycerides were considerably elevated by STZ from 3 days to the end of the experiment, which were significantly higher than those in wild-type (STZ), as shown in Fig. $1 \mathrm{~g}$ and $\mathrm{h}$. For further assessment of the significance of DPP4 activity in diabetes and dyslipidaemia, changes in DPP4 activity were monitored in the process of developing metabolic abnormalities. Plasma DPP4 activity increased progressively with time after STZ treatment in wild-type rats (Fig. 1i and j). Significant increases in plasma DPP4 activity of wild-type (STZ) were observed from $6 \mathrm{~h}$ to the end as compared with wild-type (control). Next, we investigated the correlation of plasma DPP4 activity with blood glucose or insulin in wild-type F344/Jcl rats exposed to STZ or vehicle. As a result, the degree of plasma DPP4 activity was positively correlated to the levels of blood glucose (Fig. 2a) and negatively correlated to plasma insulin
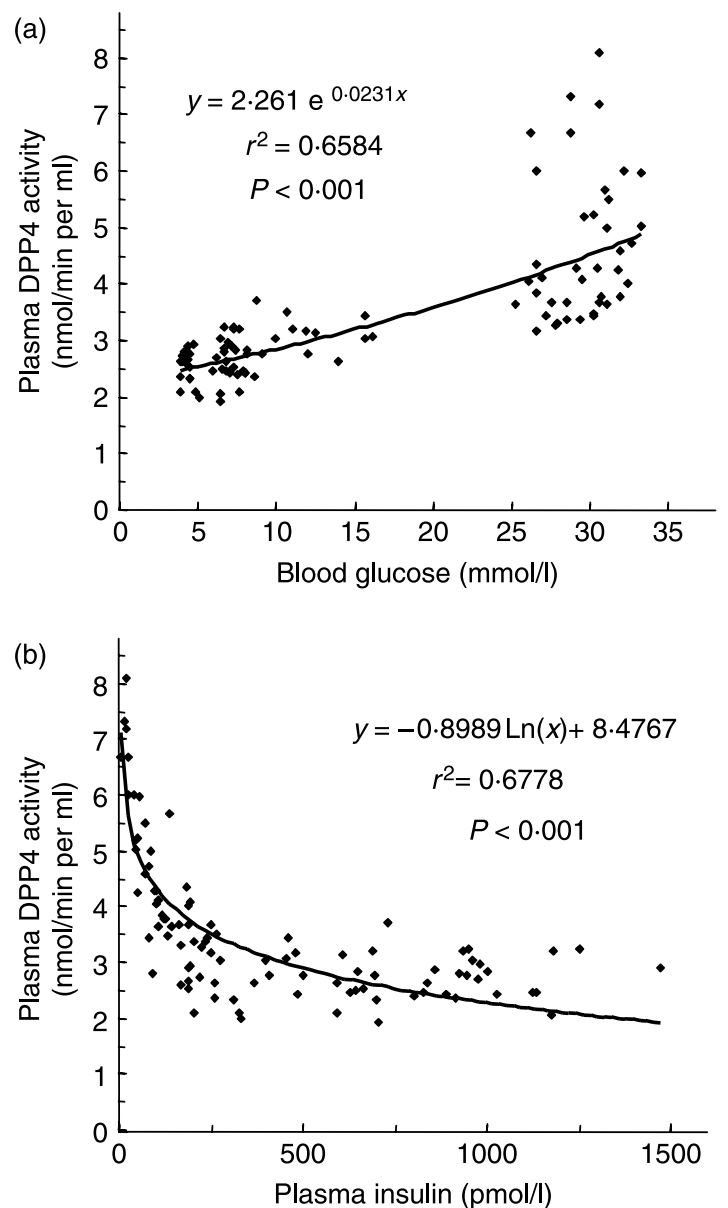

Figure 2 The correlation of plasma DPP4 activity to the levels of (a) blood glucose, (b) plasma insulin in $\mathrm{F} 344 / \mathrm{Jcl}$ (wild-type) rats treated with or without STZ $(30 \mathrm{mg} / \mathrm{kg})$. (a) $r^{2}=0.6584(P<0 \cdot 001)$; (b) $r^{2}=0.6778(P<0 \cdot 001)$. 
(a)

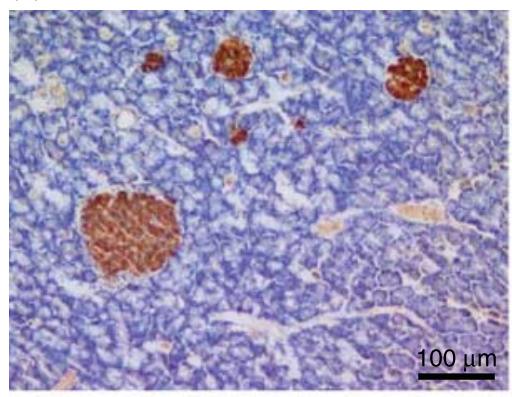

(c)

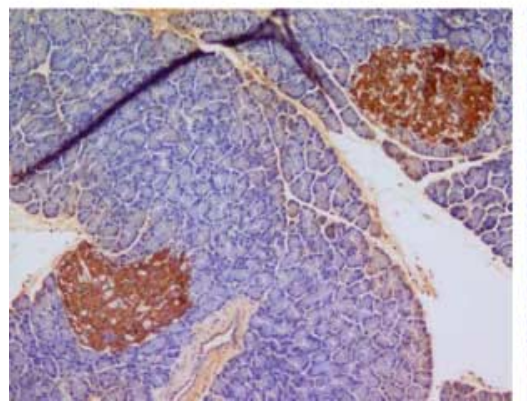

(b)

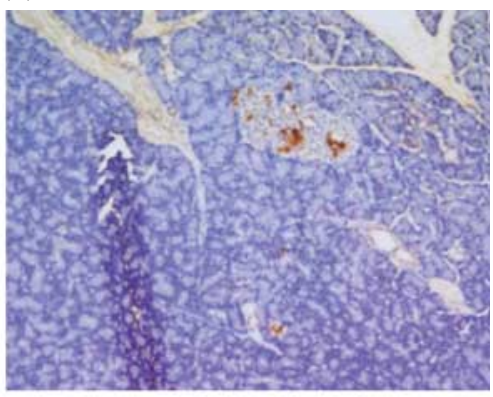

(d)

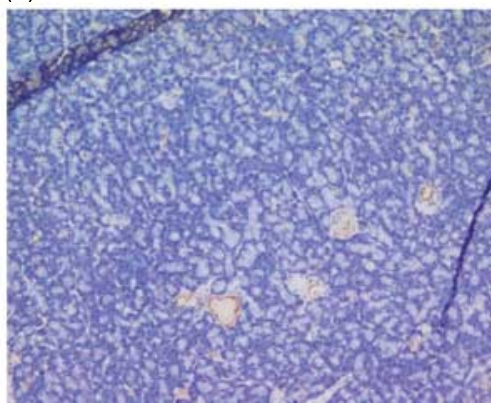

Figure 3 Histological study for insulin content of the pancreas in F344/Jcl (wild-type) and F344/DuCrlCrlj (DPP4-deficient) rats 42 days after i.p. injection with or without STZ (30 mg/kg). (a) Wild-type (control); (b) wild-type (STZ); (c) DPP4-deficient (control); (d) DPP4-deficient (STZ).

(Fig. 2b) in wild-type rats respectively. Plasma DPP4 activity in F344/DuCrlCrlj rats was not detected at any point in the experiment.

Histological and quantitative studies of insulin content in the pancreas

Insulin content in the pancreas was measured to assess the effects of DPP4 deficiency on STZ-induced islet destruction. In control groups, there were no differences in visual islet $\beta$-cell number per unit area (Fig. $3 \mathrm{a}$ and $\mathrm{c}$ ) and total pancreatic insulin content (Table 1) between wild-type and DPP4-deficient rats. At 42 days after STZ treatment, immunostained insulin was little observed in both wild-type rats (Fig. 3b) and DPP4-deficient rats (Fig. 3d). Quantitative study also showed that STZ caused a marked reduction of pancreatic insulin content in both rat strains, and STZinduced depletion of pancreatic insulin was not significantly different between these rat strains (Table 1).

Tissue weight and DPP4 expressions in tissues 42 days after $S T Z$ or vehicle treatment

There were no differences in kidney weight and epididymal fat per body weight between wild-type and DPP4-deficient rats in the control, but the liver and pancreas in DPP4-deficient rats were significantly smaller than in wild-type rats in the control (Table 2). In STZ-treated groups, the weight (g/kg body weight) of the kidney, liver and pancreas significantly increased, while the weight of epididymal fat markedly decreased as compared with control groups. DPP4 activity was detected in each tissue of wild-type rats (Table 2). DPP4 activity of the kidney significantly

Table 1 Total pancreatic insulin content 42 days after treatment with or without streptozotocin (STZ). Data are shown as the means \pm s.E.M.

\begin{tabular}{|c|c|c|c|c|}
\hline & \multicolumn{2}{|c|}{ Wild-type rats } & \multicolumn{2}{|c|}{ DPP4-deficient rats } \\
\hline & Control & STZ & Control & STZ \\
\hline $\begin{array}{l}\text { Total pancreatic insulin } \\
\text { (pmol/mg protein) }\end{array}$ & $114 \cdot 4 \pm 16 \cdot 4$ & $0 \cdot 8 \pm 0 \cdot 2^{*}$ & $107 \cdot 5 \pm 37 \cdot 0$ & $0 \cdot 7 \pm 0 \cdot 1^{+}$ \\
\hline
\end{tabular}

${ }^{*} P<0 \cdot 001$ versus wild-type (control). ${ }^{\dagger} P<0 \cdot 001$ versus DPP4-deficient (control). Wild-type (control) $n=6$; wild-type (STZ) $n=10 ;$ DPP4-deficient (control) $n=6$; DPP4-deficient (STZ) $n=10$. 
Table 2 Tissue weight and DPP4 expressions 42 days after treatment with or without streptozotocin (STZ). Data are shown as the means \pm s.E.M.

\begin{tabular}{|c|c|c|c|c|}
\hline & \multicolumn{2}{|l|}{ Wild-type rats } & \multicolumn{2}{|c|}{ DPP4-deficient rats } \\
\hline & Control & STZ & Control & STZ \\
\hline \multicolumn{5}{|c|}{ Tissue weight (g/kg body weight) } \\
\hline Liver & $36 \cdot 0 \pm 0 \cdot 5$ & $43 \cdot 3 \pm 0 \cdot 8^{\neq}$ & $30 \cdot 8 \pm 0 \cdot 5^{\mathrm{a}}$ & $40 \cdot 5 \pm 1 \cdot 0^{11}$ \\
\hline Pancreas & $2 \cdot 0 \pm 0 \cdot 2$ & $2 \cdot 8 \pm 0 \cdot 1^{+}$ & $1 \cdot 4 \pm 0 \cdot 1^{\pi}$ & $2 \cdot 6 \pm 0 \cdot 1$ \\
\hline Epididymal fat & $23 \cdot 3 \pm 1 \cdot 2$ & $5 \cdot 1 \pm 0 \cdot 8^{\neq}$ & $23 \cdot 3 \pm 0 \cdot 7$ & $6 \cdot 0 \pm 1 \cdot 3^{11}$ \\
\hline Liver & $119 \cdot 7 \pm 9 \cdot 6$ & $295 \cdot 8 \pm 58 \cdot 1^{+}$ & ND & ND \\
\hline Pancreas & $11 \cdot 2 \pm 0 \cdot 8$ & $14 \cdot 4 \pm 1 \cdot 6$ & ND & ND \\
\hline Epididymal fat & $19 \cdot 7 \pm 2 \cdot 8$ & $38 \cdot 0 \pm 2 \cdot 7^{\ddagger}$ & ND & ND \\
\hline \multicolumn{5}{|c|}{ Dpp4/Gapdh mRNA } \\
\hline Kidney & $1 \cdot 94 \pm 0 \cdot 04$ & $2 \cdot 16 \pm 0 \cdot 04^{\dagger}$ & $2 \cdot 36 \pm 0 \cdot 06^{b}$ & $2 \cdot 27 \pm 0 \cdot 05$ \\
\hline Liver & $1 \cdot 72 \pm 0 \cdot 08$ & $2 \cdot 31 \pm 0 \cdot 12^{\dagger}$ & $2 \cdot 20 \pm 0 \cdot 12^{\top}$ & $2 \cdot 46 \pm 0 \cdot 13$ \\
\hline
\end{tabular}

${ }^{*} P<0 \cdot 05,{ }^{+} P<0 \cdot 01,{ }^{\ddagger} P<0 \cdot 001$ versus wild-type (control). ${ }^{\circledR} P<0 \cdot 05, "$ P $P<0 \cdot 001$ versus DPP4-deficient (control). ${ }^{\mathrm{T}} P<0 \cdot 05,{ }^{\mathrm{a}} P<0 \cdot 01,{ }^{\mathrm{b}} P<0 \cdot 001$ versus wildtype (control). Wild-type (control) $n=6$; wild-type (STZ) $n=10$; DPP4-deficient (control) $n=6$; DPP4-deficient (STZ) $n=10$. ND, not detected.

decreased after STZ treatment in wild-type rats. By contrast, DPP4 activity of the liver and epididymal fat was significantly enhanced by STZ in wild-type rats. In F344/DuCrlCrlj rats, DPP4 activity in each tissue was not detected. On the other hand, quantitative real-time PCR showed that F344/DuCrlCrlj rats contained significantly larger amounts of Dpp 4 mRNA in the kidney and liver than in wild-type F344/Jcl rats in the control (Table 2). In STZ-treated groups, STZ significantly up-regulated $D p p 4$ mRNA expressions of the kidney and liver in F344/Jcl rats and the pancreas in both rat strains. RNA in epididymal fat could not be extracted in this experiment.

\section{Urinary parameters and renal function}

Table 3 shows the changes in urinary parameters in wild-type and DPP4-deficient rats exposed to STZ or vehicle. Urinary excretion, protein excretion and glucose excretion for $24 \mathrm{~h}$ were not different between these rat strains in the control. These parameters were significantly elevated by STZ in both wild-type and DPP4-deficient rats. There were no significant differences in these parameters between rat strains treated with STZ. DPP4 activity in urine was detected in wild-type F344/Jcl rats but not
F344/DuCrlCrlj rats. DPP4 excretion for 24 h was significantly enhanced by STZ treatment. Figure 4 shows the importance of DPP4 activity in renal function. No significant differences in serum creatinine levels were found between wild-type and DPP4-deficient rats before STZ treatment. After STZ treatment, serum creatinine levels significantly increased at 1-28 days in wild-type rats. In DPP4-deficient rats, however, serum creatinine levels were considerably elevated by STZ from 1 day to the end of the experiment, and were significantly higher than that in the wild-type (STZ), as shown in Fig. 4a. In agreement with this, creatinine clearance $(\mathrm{Ccr})$ in DPP4-deficient (STZ) was significantly lower than that that in wild-type (STZ), as shown in Fig. 4b.

\section{Discussion}

STZ is commonly used to induce type 1 and late-phase type 2 diabetic models by selective $\beta$-cell destruction in small rodents (Pospisilik et al. 2003, Srinivasan et al. 2005). In order to examine the roles of DPP4 in metabolic abnormality and renal dysfunction, we produced type 1 diabetic rats using STZ,

Table 3 Urinary parameters in wild-type and DPP4-deficient rats treated with or without streptozotocin (STZ). Data are shown as the means \pm S.E.M.

Wild-type rats

Urinary excretion $(\mathrm{ml} / 24 \mathrm{~h})$

Protein excretion $(\mathrm{mg} / 24 \mathrm{~h})$

Glucose excretion (nmol/24 h)

DPP4 excretion (nmol/min per $24 \mathrm{~h}$ )

\begin{tabular}{r} 
Control \\
\hline $5 \cdot 2 \pm 0 \cdot 9$ \\
$11 \cdot 1 \pm 1 \cdot 8$ \\
$0 \cdot 09 \pm 0 \cdot 1$ \\
$11 \cdot 3 \pm 4 \cdot 8$
\end{tabular}

DPP4-deficient rats

\begin{tabular}{llll} 
STZ & Control & & STZ \\
\cline { 1 - 1 } $97 \cdot 5 \pm 11 \cdot 2^{*}$ & $5 \cdot 8 \pm 0 \cdot 7$ & & $83 \cdot 8 \pm 8 \cdot 0^{+}$ \\
$50 \cdot 2 \pm 9 \cdot 8^{*}$ & $11 \cdot 2 \pm 1 \cdot 2$ & & $33 \cdot 9 \pm 4 \cdot 1^{+}$ \\
$12 \cdot 7 \pm 1 \cdot 9^{*}$ & $0 \cdot 08 \pm 0 \cdot 01$ & & $12 \cdot 0 \pm 1 \cdot 9^{+}$ \\
$72 \cdot 4 \pm 12 \cdot 4^{*}$ & $\mathrm{ND}$ & $\mathrm{ND}$
\end{tabular}

${ }^{*} P<0 \cdot 001$ versus wild-type (control). ${ }^{+} P<0 \cdot 001$ versus DPP4-deficient (control). Wild-type (control) $n=6$; wild-type (STZ) $n=10$; DPP4-deficient (control) $n=6$; DPP4-deficient (STZ) $n=10$. ND, not detected. 
(a)

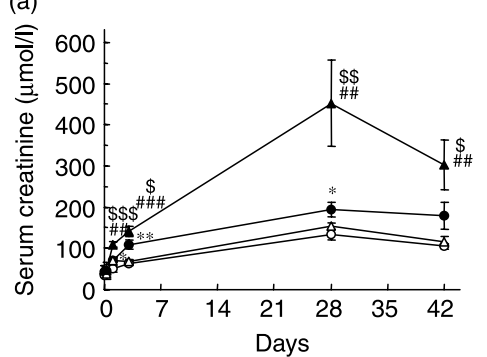

(b)

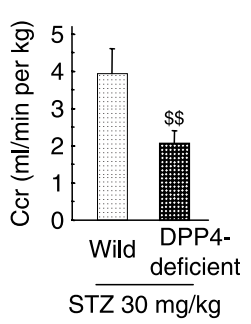

Figure 4 Interrelationship between DPP4 activity and renal function. (a) Change in serum creatinine levels in F344/JCl (wildtype) and F344/DuCrlCrlj (DPP4-deficient) rats after i.p. injection with or without STZ $(30 \mathrm{mg} / \mathrm{kg})$. Data are the means \pm s.E.M. ${ }^{*} P<0.05$ and ${ }^{* *} P<0.01$ for differences between wild-type (control) and wild-type (STZ). ${ }^{\#} P<0.01$ and ${ }^{\# \#} P<0.001$ for differences between DPP4-deficient (control) and DPP4-deficient (STZ). $\$ P<0.05$ and $\$ \$ P<0.01$ for differences between wild-type (STZ) and DPP4-deficient (STZ). Open circles: wild-type (control) $n=6$; closed circles: wild-type (STZ) $n=10$; open triangles: DPP4deficient (control) $n=6$; closed triangles: DPP4-deficient (STZ) $n=10$. (b) Difference in creatinine clearance (Ccr) between F344/JCl (wild-type) and F344/DuCrlCrlj (DPP4-deficient) rats treated with STZ $(30 \mathrm{mg} / \mathrm{kg}) .{ }^{\$} P<0 \cdot 01$ for difference between wildtype (STZ) and DPP4-deficient (STZ) $n=10$.

with extensive islet destruction and depletion of both pancreatic and plasma insulin. In STZ-induced diabetic rats, dyslipidaemia and marked loss of adipose tissue mass, which are characteristics of insulin insufficiency, were also observed. Moreover, it was confirmed that STZ enhanced both expressions of Dpp 4 mRNA in all examined tissues and circulating DPP4 enzyme activity in wild-type rats, showing a positive correlation of plasma DPP4 activity to blood glucose. The main source of endogenous DPP4 has been proposed to be microvascular endothelial cells of some areas, including the kidney (Augustyns et al. 1999, Pala et al. 2003). An in vitro study determined that exposure to high glucose caused increases in Dpp 4 mRNA and enzyme activity in human glomerular endothelial cells (Pala et al. 2003). From these findings, it is suggested that the STZ-induced increase in plasma DPP4 activity is attributed to enhanced biosynthesis of DPP4 enzyme and its secretion in endothelial cells by high blood glucose. It may also be reasonable that hyperglycaemia-induced cell damage, including the kidney, caused DPP4 to leak into the circulation, since kidney DPP4 activity was reduced even though kidney Dpp 4 mRNA was up-regulated in STZinduced diabetes. In addition, the increased activity of plasma DPP4 could still worsen hyperglycaemia since DPP4 activation may lead to decreases in the anti-diabetic effects of GLP-1 and GIP; therefore, it was supposed that DPP4 inhibition was effective in ameliorating STZ-induced metabolic abnormalities. A DPP4 inhibitor, P32/98, was reported to reduce the progressive severity of diabetes induced by STZ in Wistar rats, considering that DPP4 inhibition followed by increased GLP-1 and GIP prevented insulin-secretory capacity via enhanced islet neogenesis, $\beta$-cell survival and insulin biosynthesis (Pospisilik et al. 2003). Consistent with this, mice lacking the gene encoding DPP4 were refractory to loss of $\beta$-cell mass and hyperglycaemia evoked by high-fat diet for 8 weeks followed by STZ injection (Conarello et al. 2003). In the present study, DPP4-deficient rats exhibited significantly lower levels of blood glucose and food intake than wild-type rats in the early phase after STZ treatment, even though STZ-induced insulin depletion occurred in DPP4deficient rats as well as wild-type rats. The failure of DPP4 deficiency to prevent $\beta$-cell function from STZ exposure could be attributed to the severe islet destruction and depletion of pancreatic insulin in this experiment. In addition, in control groups, the levels of plasma insulin in DPP4-deficient rats were significantly lower than that in wild-type rats after feeding, which could indicate that DPP4-deficient rats were more sensitive to insulin than wild-type rats. Accordingly, the present study suggests that DPP4-deficient rats are resistant to developing hyperglycaemia owing to lower food intake and higher insulin sensitivity.

Contrary to our expectations, greater increases in circulating cholesterol and triglycerides were found in DPP4-deficient rats than wild-type rats after STZ treatment. These results imply that DPP4-deficient rats are resistant to developing hyperglycaemia, but susceptible to dyslipidaemia induced by STZ. Recent studies of obesity have proposed that GIP, a substrate of DPP4, plays an important role in lipid abnormality since GIP is a key element linking overnutrition to obesity through increased nutrient uptake and triglyceride accumulation in adipocytes (Miyawaki et al. 2002, McClean et al. 2007). However, the mechanisms underlying excessive increases in circulating lipid by deficiency of DPP4 activity are unknown in non-obese diabetes. In wild-type rats, hypertriglyceridaemia occurred significantly in only the early phase (1-3 days) after STZ treatment; thus, the elevation of triglycerides appears to be attenuated by increased plasma DPP4 activity after STZ treatment. Furthermore, STZ also enhanced liver DPP4 activity and mRNA expression. Although additional studies characterizing the roles of DPP4 activity in the liver or small intestine in lipid transport, biosynthesis and secretion are needed, DPP4 activation may enable lipid metabolism in STZ-induced non-obese diabetes.

The importance of DPP4 in renal function was assessed in this model. As a result, decreased DPP4 enzyme activity and increased Dpp 4 mRNA expression were detected in the kidney of wild-type rats after STZ treatment. Moreover, the ratio of kidney weight to body weight, serum creatinine levels and excreted amounts of urinary protein, glucose and DPP4 enzyme were significantly enhanced by STZ. Increases in kidney weight, serum creatinine and urinary excretion of protein and glucose have been detected in clinical and experimental diabetic nephropathy. The reasons for decreased kidney DPP4 activity and increased urinary DPP4 excretion by STZ remain uncertain; however, they may occur as a result of renal damage, which causes the leakage of microvascular endothelial DPP4 enzyme of the kidney, as suggested by previous reports (Scherberich et al. 1992, Augustyns et al. 1999, Pala et al. 2003). Of note, this comparative study of wild-type 
and DPP4-deficient rats determined that DPP4-deficient rats were more sensitive to GFR reduction induced by STZ. This finding indicates that DPP4 activity in plasma and/or kidney may be closely related to protection against renal dysfunction. DPP4 is most expressed in the kidney; however, the role of DPP4 activity in renal function is poorly understood. A recent study reported that the inhibition of DPP4 activity in kidney augmented the ability of endogenous neuropeptide $\mathrm{Y}_{1-36}$ released form renal sympathetic nerves to enhance angiotensin II-mediated renal vasoconstriction in vitro, since neuropeptide $\mathrm{Y}_{1-36}$ is a substrate for DPP4 (Jackson \& Mi 2008). This could explain the reduction in GFR in DPP4-deficient rats. Another possibility is that dyslipidaemia is one of the risk factors of glomerular sclerosis, proteinurea and GFR reduction (Molitch 2006, Fogo 2007). In the present study, however, the time course of serum creatinine shows that the GFR reduction in DPP4-deficient rats occurred rapidly (within days) after STZ treatment. Therefore, it is unlikely that the increased circulating lipid in DPP4-deficient rats could alter renal function within this time frame. Moreover, there were no significant differences in urinary protein excretion and kidney weight (renal hypertrophy) between wild-type and DPP4-deficient rats after STZ treatment. Considering all of the above, it is strongly presumed that the adverse effect of DPP4 deficiency on the kidney is functional rather than structural. However, it is possible that a long-term blockade of DPP4 accompanied by chronic dyslipidaemic state will lead to renal failure structurally as well as functionally. In addition, chronic renal disease, especially with nephrotic syndrome, has been reported to develop dyslipidaemia (Farbakhsh \& Kasiske 2005, Molitch 2006), which suggests that a close relationship exists among dyslipidaemia, renal dysfunction and DPP4 in late-stage diabetes, but further studies are required.

In conclusion, DPP4 deficiency delayed the onset of diabetes, but worsened dyslipidaemia and renal dysfunction induced by STZ. This study could be the basis of additional research into the advantages and disadvantages of DPP4 inhibition for insulin-dependent diabetes.

\section{Declaration of interest}

The authors declare that there is no conflict of interest that would prejudice the impartiality of this scientific work.

\section{Funding}

This work was supported in part by Grants-in-Aid for Scientific Research on Priority Areas from the Ministry of Education, Culture, Sports, Science and Technology of Japan.

\section{Acknowledgements}

The authors wish to thank Dr Ashraf A. Ewis for the English language editing and helpful discussions.

\section{References}

Augustyns K, Bal G, Thonus G, Belyaev A, Zhang XM, Bollaert W, Lambeir AM, Durinx C, Goossens F \& Haemers A 1999 The unique properties of dipeptidyl-peptidase IV (DPP IV/CD26) and the therapeutic potential of DPP IV inhibitors. Current Medicinal Chemistry 6 311-327.

Conarello SL, Li ZH, Ronan J, Roy RS, Zhu L, Jiang GQ, Liu F, Woods J, Zycband E, Moller DE et al. 2003 Mice lacking dipeptidyl peptidase IV are protected against obesity and insulin resistance. PNAS $1006825-6830$.

Deacon CF, Wamberg S, Bie P, Hughes TE \& Holst JJ 2002 Preservation of active incretin hormones by inhibition of dipeptidyl peptidase IV suppresses meal-induced incretin secretion in dogs. Journal of Endocrinology 172 355-362.

Durinx C, Neels H, Van der Auwera JC, Naelaerts K, Scharpe S \& De Meester I 2001 Reference values for plasma dipeptidyl-peptidase IV activity and their association with other laboratory parameters. Clinical Chemistry and Laboratory Medicine 39 155-159.

Erickson RH, Suzuki Y, Sedlmayer A \& Kim YS 1992 Biosynthesis and degradation of altered immature forms of intestinal dipeptidyl peptidase IV in a rat strain lacking the enzyme. Journal of Biological Chemistry 267 21623-21629.

Farbakhsh K \& Kasiske BL 2005 Dyslipidemias in patients who have chronic kidney disease. Medical Clinics of North America 89 689-699.

Flock G, Baggio LL, Longuet C \& Drucker DJ 2007 Incretin receptors for glucagon-like peptide 1 and glucose-dependent insulinotropic polypeptide are essential for the sustained metabolic actions of vildagliptin in mice. Diabetes 56 3006-3013.

Fogo AB 2007 Mechanisms of progression of chronic kidney disease. Pediatric Nephrology 22 2011-2022.

Fonseca V, Schweizer A, Albrecht D, Baron MA, Chang I \& Dejager S 2007 Addition of vildagliptin to insulin improves glycaemic control in type 2 diabetes. Diabetologia 50 1148-1155.

Green BD, Gault VA, O'Harte FPM \& Flatt PR 2004a Structurally modified analogues of glucagon-like peptide-1 (GLP-1) and glucose-dependent insulinotropic polypeptide (GIP) as future antidiabetic agents. Current Pharmaceutical Design 10 3651-3662.

Green BD, Mooney MH, Gault VA, Irwin N, Bailey CJ, Harriott P, Greer B, O'Harte FPM \& Flatt PR 2004b N-terminal His (7)-modification of glucagon-like peptide-1 (7-36) amide generates dipeptidyl peptidase IV-stable analogues with potent antihyperglycaemic activity. Journal of Endocrinology 180 379-388.

Green BD, Irwin N, Duffy NA, Gault VA, O'Harte FPM \& Flatt PR 2006 Inhibition of dipeptidyl peptidase-IV activity by metformin enhances the antidiabetic effects of glucagon-like peptide-1. European Journal of Pharmacology 547 192-199.

Holst JJ 2006 Glucagon-like peptide-1: from extract to agent. The Claude Bernard Lecture, 2005. Diabetologia 49 253-260.

Iwakura H, Hosoda K, Son C, Fujikura J, Tomita T, Noguchi M, Ariyasu H, Takaya K, Masuzaki H, Ogawa Y et al. 2005 Analysis of rat insulin II promoter-ghrelin transgenic mice and rat glucagon promoter-ghrelin transgenic mice. Journal of Biological Chemistry 280 15247-15256.

Jackson EK \& Mi Z 2008 Sitagliptin augments sympathetic enhancement of the renovascular effects of angiotensin II in genetic hypertension. Hypertension 51 1637-1642.

Lenhard JM, Croom DK \& Minnick DT 2004 Reduced serum dipeptidyl peptidase-IV after metformin and pioglitazone treatments. Biochemical and Biophysical Research Communications 324 92-97.

Lindsay JR, Duffy NA, McKillop AM, Ardill J, O'Harte FPM, Flatt PR \& Bell PM 2005 Inhibition of dipeptidyl peptidase IV activity by oral metformin in Type 2 diabetes. Diabetic Medicine 22 654-657.

Lugari R, Dei Cas A, Ugolotti D, Barilli AL, Camellini C, Ganzerla GC, Luciani A, Salerni B, Mittenperger F, Nodari S et al. 2004 Glucagon-like peptide 1 (GLP-1) secretion and plasma dipeptidyl peptidase IV (DPP-IV) activity in morbidly obese patients undergoing biliopancreatic diversion. Hormone and Metabolic Research 36 111-115. 
Mannucci E, Pala L, Ciani S, Bardini G, Pezzatini A, Sposato I, Cremasco F, Ognibene A \& Rotella C 2005 Hyperglycaemia increases dipeptidyl peptidase IV activity in diabetes mellitus. Diabetologia 48 1168-1172.

McClean PL, Irwin N, Cassidy RS, Holst JJ, Gault VA \& Flatt PR 2007 GIP receptor antagonism reverses obesity, insulin resistance, and associated metabolic disturbances induced in mice by prolonged consumption of high-fat diet. American Journal of Physiology. Endocrinology and Metabolism 293 E1746-E1755

McKillop AM, Duffy NA, Lindsay JR, O'Harte FPM, Bell PM \& Flatt PR 2008 Decreased dipeptidyl peptidase-IV activity and glucagon-like peptide1 (7-36) amide degradation in type 2 diabetic subjects. Diabetes Research and Clinical Practice 79 79-85.

De Meester I, Durinx C, Bal G, Proost P, Struyf S, Goossens F, Augustyns K \& Scharpe S 2000 Natural substrates of dipeptidyl peptidase IV. Advances in Experimental Medicine and Biology 477 67-87.

Meneilly GS, Demuth HU, McIntosh CHS \& Pederson RA 2000 Effect of ageing and diabetes on glucose-dependent insulinotropic polypeptide and dipeptidyl peptidase IV responses to oral glucose. Diabetic Medicine 17346-350.

Mentlein R 1999 Dipeptidyl-peptidase IV (CD26)-role in the inactivation of regulatory peptides. Regulatory Peptides 85 9-24.

Mikhail N 2008 Incretin mimetics and dipeptidyl peptidase 4 inhibitors in clinical trials for the treatment of type 2 diabetes. Expert Opinion on Investigational Drugs 17 845-853.

Mitani H, Takimoto M, Hughes TE \& Kimura M 2002 Dipeptidyl peptidase IV inhibition improves impaired glucose tolerance in high-fat diet-fed rats: study using a Fischer 344 rat substrain deficient in its enzyme activity. Japanese Journal of Pharmacology 88 442-450.

Miyawaki K, Yamada Y, Ban N, Ihara Y, Tsukiyama K, Zhou HY, Fujimoto S, Oku A, Tsuda K, Toyokuni S et al. 2002 Inhibition of gastric inhibitory polypeptide signaling prevents obesity. Nature Medicine 8 738-742.

Molitch ME 2006 Management of dyslipidemias in patients with diabetes and chronic kidney disease. Clinical Journal of the American Society of Nephrology 1 1090-1099.

Pala L, Mannucci E, Pezzatini A, Ciani S, Sardi J, Raimondi L, Ognibene A, Cappadona A, Vannelli BG \& Rotella CM 2003 Dipeptidyl peptidase-IV expression and activity in human glomerular endothelial cells. Biochemical and Biophysical Research Communications 310 28-31.

Pospisilik JA, Martin J, Doty T, Ehses JA, Pamir N, Lynn FC, Piteau S, Demuth HU, McIntosh CHS \& Pederson RA 2003 Dipeptidyl peptidase IV inhibitor treatment stimulates beta-cell survival and islet neogenesis in streptozotocin-induced diabetic rats. Diabetes $\mathbf{5 2}$ 741-750.
Ryskjaer J, Deacon CF, Carr RD, Krarup T, Madsbad S, Holst J \& Vilsboll T 2006 Plasma dipeptidyl peptidase-IV activity in patients with type-2 diabetes mellitus correlates positively with HbAlc levels, but is not acutely affected by food intake. European Journal of Endocrinology 155 485-493.

Scherberich JE, Wiemer J \& Schoeppe W 1992 Biochemical and immunological properties of urinary angiotensinase A and dipeptidylaminopeptidase IV - their use as markers in patients with renal cell injury. European Journal of Clinical Chemistry and Clinical Biochemistry 30 663-668.

Srinivasan K, Viswanad B, Asrat L, Kaul CL \& Ramarao P 2005 Combination of high-fat diet-fed and low-dose streptozotocin-treated rat: a model for type 2 diabetes and pharmacological screening. Pharmacological Research $\mathbf{5 2}$ 313-320.

Thompson NL, Hixson DC, Callanan H, Panzica M, Flanagan D, Faris RA, Hong WJ, Hartel-Schenk S \& Doyle D 1991 A Fischer rat substrain deficient in dipeptidyl peptidase IV activity makes normal steady-state RNA levels and an altered protein - use as a liver-cell transplantation model. Biochemical Journal 273 497-502.

Villhauer EB, Brinkman JA, Naderi GB, Burkey BF, Dunning BE, Prasad K, Mangold BL, Russell ME \& Hughes TE 2003 1-[[(3-hydroxy-1-adamantyl) amino]acetyl]-2-cyano-(S)-pyrrolidine: a potent, selective, and orally bioavailable dipeptidyl peptidase IV inhibitor with antihyperglycemic properties. Journal of Medicinal Chemistry 46 2774-2789.

Yasuda N, Nagakura T, Yamazaki K, Inoue T \& Tanaka I 2002 Improvement of high fat-diet-induced insulin resistance in dipeptidyl peptidase IV-deficient Fischer rats. Life Sciences 71 227-238.

Zhou YP, Marlen K, Palma JF, Schweitzer A, Reilly L, Gregoire FM, Xu GG, Blume JE \& Johnson JD 2003 Overexpression of repressive cAMP response element modulators in high glucose and fatty acid-treated rat islets - a common mechanism for glucose toxicity and lipotoxicity? Journal of Biological Chemistry 278 51316-51323.

Received in final form 8 October 2008 Accepted 15 October 2008 Made available online as an Accepted Preprint 17 October 2008 